\title{
Fuzzy Comprehensive Evaluation of Oil Field Waterflooding Effect
}

\author{
Wei Xiao, Yongshun Liang \\ Faculty of Science, Nanjing University of Science and Technology, Nanjing, China \\ Email: xiaowei@njust.edu.cn
}

How to cite this paper: Xiao, W. and Liang, Y.S. (2021) Fuzzy Comprehensive Evaluation of Oil Field Waterflooding Effect. International Journal of Geosciences, 12, 560-571.

https://doi.org/10.4236/ijg.2021.126031

Received: March 25, 2021

Accepted: June 15, 2021

Published: June 18, 2021

Copyright $\odot 2021$ by author(s) and Scientific Research Publishing Inc. This work is licensed under the Creative Commons Attribution International License (CC BY 4.0).

http://creativecommons.org/licenses/by/4.0/

(c) (i) Open Access

\begin{abstract}
Oil field waterflooding is a complex man-controlled systematic behavior, and the related evaluation methods vary greatly. This paper put forward a fuzzy comprehensive method of evaluating controlled development level by analysis of the macroscopic evaluation to oil field waterflooding effect with combination of original reservoir geological state. This fuzzy evaluation technique bears unique advantages because there is little difference among evaluation indexes which represent the dynamic and static state of regional neighborhood of development units (blocks, Production Company, etc.). Not only the mathematical method for evaluating oil field waterflooding effect is set up, but also the method is applied in three blocks of $\mathrm{D}$ oil field. The calculated results show the effectiveness and practicability of the method.
\end{abstract}

\section{Keywords}

Comprehensive Evaluation, Oil Field Waterflooding, Development Effect, Fuzzy Mathematics

\section{Introduction}

Waterflooding as the dominant technology in oil field development is facing a universal problem of associated high water cut and how to improve the waterflooding efficiency during high water cut stage has become a highlight for all the researchers in the world. Many of the oil fields in China have entered extra-high water cut stage after several decades of development, which are now encountered by the conjuncture of intractable remaining oil exploitation, deficient reserve replacements and continuous production declination. Reasonable and proper development effect evaluation would help to determine the direction for remaining oil exploitation and adjustment and thus guiding more effective and scientific reservoir development, as well as providing reference to the composi- 
tion of development plans and long-term decisions. The essence of evaluating the operational performance of any system is to judge the scientism, rationality and optimality of the system. Comprehensive evaluation of the operational performance of a system should set off from the perspective of systematology. Judging from the above point of view, during development effect evaluation, the first step is to find out evaluation indexes [1] [2]. This paper just applies these evaluation indexes, and focuses on comprehensive techniques.

Many of the development effect evaluation methods usually lean on oil production mechanism [3]-[14], however development effect of water flooding is a cumulative, macroscopic, integral and systematical issue [15] [16] that requires evaluation from the perspective of systematology [17] [18]. Although considering the development effect from the perspective of oil production mechanism could seize the key point and provide detailed operational ways for production adjustment, this method is restricted by the short coming of partial evaluation.

On the other side, many single indexe [6] [14] evaluation or correlation evaluation of practical curves and theoretical curves (usually 2D and two indexes) [7] [13] are confined. The theory of systematology [3] [8] should be adopted in the analysis of injection and production correlations in partial well clusters which have undergone water flooding, in order to ascertain the efficiency of water flooding and the utility of water flooding adjustment and plan. The limitation of this method is that it is only fit for partial well clusters and the range of the evaluation unit is too small which is not advisable to apply to the whole block or even an oil field. Evaluating water flooding effect from the perspective of systematology is discussed in this paper. Because of the slight differential of evaluation attributes caused by geographic proximity of the evaluated units, we would choose fuzzy mathematics methods [19] to handle it. Fuzzy remarks range is

$$
V=\left\{\begin{array}{lll}
h & z & c
\end{array}\right\}=\{\text { good normal poor }\} \text {. }
$$

The universe of ambiguity factors could be defined as two aspects, first, factors that decide the original geologic property of the reservoir, and second, factors that dominate the development level of the oil field. To determine whether the water flooding effect is good or not mainly depends on the amount of oil production, which if explained from systematology is mainly controlled by two attributes, one is original reservoir geologic property and the other is controlled development level. Original reservoir geologic property refers to the natural oil productivity of the reservoir, while the controlled development level refers to treatments taken during water flooding process, including water injection pattern, well array, perforation, fracturing and acid treating, etc., some reservoirs with predominance natural oil productivity probably would not obtain satisfactory crude oil production after exploration, only by scientific and rational development control could the maximum crude oil recovery rate be reached. This is the purpose of comprehensive evaluation, to see if the reservoirs with different natural oil productivity are explored by reasonable development control and if different reservoirs are matched with corresponding development treatments. 
Water flooding effect evaluation would cover evaluations on both original reservoir geologic property and controlled development level and in addition the overall effects based on the former two evaluations.

Contexture of this paper follows the contents of regular comprehensive evaluation, first building an evaluation index system, and then calculating the evaluation index weight, successively, setting up unified standard function with fuzzy membership degree for each evaluation index, and finally the comprehensive evaluation.

\section{Comprehensive Evaluation Index Set}

Through system theory and control theory analysis, the structure of systematic behavior of oil field waterflooding can be found out. Water well system is defined as the group composed of all water wells, oil well system is defined as the group composed of all oil wells.

The index system of original reservoir property is the information indicator of reservoir system, while the index system of controlled development level is the information indicator of oil and water well system. Based on comprehensive evaluation of the oil field reality (D oil field), through oil field development engineering mechanism, the index system of the two oil field development attributes can be defined as follows by using quantitative and qualitative analysis (multiple correlation analysis).

All these indexes can be screen out as follows, the details are referenced to [1] [2].

Factors for evaluating original reservoir geological property include:

1) channel sand proportion $R_{h d}$;

2) effective permeability $K_{e}$;

3) net thickness $h_{e}$;

4) coefficient of permeability variation $V_{K}$;

5) initial oil saturation $S_{o}$;

6) oil viscosity $\mu_{o}$;

7) difference between reservoir pressure and saturation pressure $P_{b}$;

8) reserves abundance $A_{q}$;

9) displacement efficiency $\eta_{D}$.

Factors for evaluating controlled development level include:

1) total pressure difference $\Delta P$;

2) oil and water well ratio $R_{j}$;

3) well spacing density $A_{j}$;

4) cumulative water storage rate $\gamma_{w}$;

5) cumulative injection production ratio $R_{r c}$;

6) waterflooding controlled degree $C_{h}$;

7) reserves recovery degree $D_{h}$.

\section{Calculation about Index Weight}

For comprehensive evaluation to any complex system, it is not easy to determine 
the index weight rationally after drawing up evaluation index system. For the systematic behavior of evaluation, if there are too many attributes index (for example, 16 indexes for waterflooding effect evaluation), only mathematic methods can be used to determine the weight. But this kind of quantitative method relies greatly on the samples need to be evaluated. There are three blocks (samples) in this D oil field. According to oil field waterflooding system attributes, the multiple correlation coefficient and variation coefficient are used to calculate index weight in this paper. Then, the final weight is got by geometric averaging the two weights.

\subsection{Index Weight of Reservoir Geological Status}

Assume that the multiple correlation coefficients with $m$ indexes are $\rho_{1}, \rho_{2}, \cdots, \rho_{m}$, then the number $k$ coefficient reflects the ability of all the other indexes except $k$ index to replace $k$ index. Hence, the larger the $k$ index, the smaller its function, so the reciprocal value of multiple correlation coefficient can be taken as weight. Then the weight formula for multiple correlation coefficients is developed,

$$
v_{k}^{1}=\frac{\prod_{i=1}^{8} \rho_{i}^{2}}{\rho_{k}^{2} \sum_{i=1}^{8} \prod_{j \neq i, j=1}^{8} \rho_{j}^{2}}(k=1,2, \cdots, m)
$$

Multiple correlation coefficient can be calculated from data in Table 1 (all the subscription number is in accordance with the index sequence in factor range).

The weight is calculated to be:

$$
V^{1}=\left(v_{1}^{1}, v_{2}^{1}, \cdots, v_{9}^{1}\right)^{\mathrm{T}}=(0.172,0.105,0.088,0.123,0.101,0.098,0.088,0.118)^{\mathrm{T}}
$$

In addition, from statistical theory we can know that, the larger the variance of each index, the stronger the corresponding attributes of each index for evaluating the targets, the more attention should be paid to this index, thus the larger of this index weight. This is the so called theory of determining index weight by variation coefficient.

As above mentioned, by using data in Table 4, weight can be obtained by variation coefficient calculating method [15].

$$
\begin{aligned}
V^{2} & =\left(v_{1}^{2}, v_{2}^{2}, \cdots, v_{9}^{2}\right)^{\mathrm{T}} \\
& =(0.102,0.081,0.186,0.053,0.026,0.067,0.221,0.216,0.048)^{\mathrm{T}}
\end{aligned}
$$

In order to avoid the unilateralism of different methods for calculating weights, comprehensive weight is obtained by geometric averaging the weights obtained from the two above methods.

Table 1. Multiple correlation coefficients of geological statue index system.

\begin{tabular}{ccccccccc}
\hline$\rho_{1}^{2}$ & $\rho_{2}^{2}$ & $\rho_{3}^{2}$ & $\rho_{4}^{2}$ & $\rho_{5}^{2}$ & $\rho_{6}^{2}$ & $\rho_{7}^{2}$ & $\rho_{8}^{2}$ & $\rho_{9}^{2}$ \\
\hline 0.172 & 0.105 & 0.088 & 0.123 & 0.106 & 0.101 & 0.098 & 0.088 & 0.118 \\
\hline
\end{tabular}


Formula for geometric average is:

$$
V=\sqrt{V^{1} \circ V^{2}}
$$

i.e.

$$
v_{k}=\sqrt{v_{k}^{1} v_{k}^{2}}, k=1,2, \cdots, 9
$$

Comprehensive weight is obtained from the above formula

$$
\begin{aligned}
V & =\left(v_{1}, v_{2}, \cdots, v_{9}\right)^{\mathrm{T}} \\
& =(0.1428,0.0993,0.1377,0.0874,0.0567,0.0888,0.1583,0.1484,0.0806)
\end{aligned}
$$

In order to show the relationship between the indexes weight with the index itself, these data is tabularized in Table 2.

\subsection{Controlling Development Index Weight}

Based on the same principle, the weight of control index can be obtained. The following formula (3.4)-(3.6) are weight from multiple correlation coefficient, weight from variation coefficient and comprehensive weight from geometric average respectively.

$$
\begin{gathered}
W^{1}=\left(w_{1}^{1}, w_{2}^{1}, \cdots, w_{7}^{1}\right)^{\mathrm{T}}=(0.158,0.208,0.146,0.117,0.128,0.126,0.117)^{\mathrm{T}} \\
\begin{aligned}
W^{2}=\left(w_{1}^{2}, w_{2}^{2}, \cdots, w_{7}^{2}\right)^{\mathrm{T}}=(0.315,0.102,0.248,0.148,0.073,0.039,0.039)^{\mathrm{T}} \\
W= \\
W^{1} \circ W^{2}=\left(w_{1}, w_{2}, \cdots, w_{7}\right)^{\mathrm{T}} \\
=(0.109,0.195,0.087,0.121,0.124,0.151,0.132,0.08)^{\mathrm{T}}
\end{aligned}
\end{gathered}
$$

These data is tabularized in Table 3.

Table 2. Comprehensive weight of geological index.

\begin{tabular}{c|c|c|c|c}
\hline $\begin{array}{c}\text { Channel sand } \\
\text { proportion }\end{array}$ & Effective permeability & Net thickness & $\begin{array}{c}\text { Permeability } \\
\text { variation coefficient }\end{array}$ & Oil saturation \\
\hline 0.1428 & 0.0993 & 0.1377 & 0.0874 & 0.0567 \\
\hline $\begin{array}{c}\text { Petroleum } \\
\text { viscosity }\end{array}$ & $\begin{array}{c}\text { Difference between } \\
\text { reservoir pressure } \\
\text { and saturation pressure }\end{array}$ & $\begin{array}{c}\text { Reserves } \\
\text { abybdabce }\end{array}$ & $\begin{array}{c}\text { Displacement } \\
\text { efficiengcy }\end{array}$ & \\
\hline 0.0888 & 0.1583 & 0.1484 & 0.0806 & \\
\hline
\end{tabular}

Table 3. Controll index weight.

\begin{tabular}{c|c|c|c|c}
\hline Controll index & $\begin{array}{c}\text { Total pressure } \\
\text { difference }\end{array}$ & $\begin{array}{c}\text { Oil and water } \\
\text { well ratio }\end{array}$ & Well spacing density & $\begin{array}{c}\text { Cumulative } \\
\text { water storage rate }\end{array}$ \\
\hline weight & 0.2516 & 0.1553 & 0.2028 & 0.1404 \\
\hline Controll index & $\begin{array}{c}\text { Cumulative injectio } \\
\text { production ratio }\end{array}$ & $\begin{array}{c}\text { Waterflooding } \\
\text { controlling degree }\end{array}$ & $\begin{array}{c}\text { Reserve } \\
\text { producing degree }\end{array}$ & \\
\hline weight & 0.103 & 0.0748 & 0.0722 & \\
\hline
\end{tabular}




\section{Establishment of Fuzzy Membership Function}

A unified evaluation standard is needed in order to rationally evaluate each index; this is the function with fuzzy membership degree of each index. Based on characteristic analysis of 9 geological indexes and 7 controlling indexes, it is found that the comprehensive evaluation index can be classified into two types: one is tendency index, the other is moderate index.

For tendency index, there are two situations during evaluation: one is, the larger the index value, the larger the evaluation value (abbreviated as the larger the better); another one is, the larger the index value, the smaller the evaluation value (abbreviated as the smaller the better). Thus there are only two types of functions with fuzzy membership degree for tendency index. In this situation, assume the evaluation values corresponding to fuzzy remarks range

$$
V=\left\{\begin{array}{lll}
h & z & c
\end{array}\right\}=\{\text { good normal poor }\}
$$

are $x_{g}, x_{n}, x_{b}$ respectively (subscript $g$ stands for good, $n$ for normal, $b$ for poor).

For moderate index, it would be more complex when setting up corresponding function with fuzzy membership degree. First, the "moderate value $x_{o}$ " ( $o$ stand for optimization) of moderate index should be calculated, or to be called "optimized as rational value". Then set up function with fuzzy membership degree $x^{*}=x / x_{0}$. Apparently, now the more close of $x^{*}$ to 1 , the larger the evaluation value, its standards values of "good, normal, poor" are all symmetrical to 1 . In this situation, assume the evaluation values corresponding to fuzzy range

$$
V=\left\{\begin{array}{lll}
h & z & c
\end{array}\right\}=\{\text { good normal poor }\}
$$

are $x_{g}^{+1}, x_{g}^{-1}, x_{n}^{+1}, x_{n}^{-1}, x_{b}^{+1}, x_{b}^{-1}$ respectively, among them, superscript +1 stands for points larger than $1,-1$ stands for points smaller than 1 .

Based on above analysis, the broken line function with fuzzy membership degree of three types of index can be set up.

- Function with fuzzy membership degree of the larger the better index $x$

$$
\begin{gathered}
\mu_{h}(x)=\left\{\begin{array}{lr}
0, & x \leq x_{n} \\
\frac{x-x_{n}}{x_{g}-x_{n}}, & x_{n}<x<x_{g} \\
1, & x \geq x_{g}
\end{array}\right. \\
\mu_{z}(x)=\left\{\begin{array}{lc}
0, & x \leq x_{b} \\
\frac{x-x_{b}}{x_{n}-x_{b}}, & x_{b}<x \leq x_{n} \\
\frac{x_{g}-x}{x_{g}-x_{n}}, & x_{n}<x \leq x_{g} \\
0, & x \geq x_{g}
\end{array}\right.
\end{gathered}
$$




$$
\mu_{c}(x)=\left\{\begin{array}{lc}
1, & x \leq x_{b} \\
\frac{x_{n}-x}{x_{n}-x_{b}}, & x_{b}<x<x_{n} \\
0, & x \geq x_{n}
\end{array}\right.
$$

- Function with fuzzy membership degree of the smaller the better index $x$

$$
\begin{aligned}
& \mu_{h}(x)=\left\{\begin{array}{lc}
1, & x \leq x_{b} \\
\frac{x_{n}-x}{x_{n}-x_{b}}, & x_{b}<x \leq x_{n} \\
0, & x>x_{n}
\end{array}\right. \\
& \mu_{z}(x)= \begin{cases}0, & x \leq x_{b} \\
\frac{x-x_{b}}{x_{n}-x_{b}}, & x_{b}<x \leq x_{n} \\
\frac{x_{g}-x}{x_{g}-x_{n}}, & x_{n}<x \leq x_{g} \\
0, & x>x_{g}\end{cases} \\
& \mu_{c}(x)= \begin{cases}0, & x \leq x_{n} \\
\frac{x-x_{n}}{x_{g}-x_{n}}, & x_{n}<x \leq x_{g} \\
1, & x>x_{g}\end{cases}
\end{aligned}
$$

- Function with fuzzy membership degree of moderate index $x^{*}$

$$
\begin{gathered}
\mu_{h}\left(x^{*}\right)=\left\{\begin{array}{lc}
0, & x^{*} \leq x_{n}^{-1} \\
\frac{x^{*}-x_{n}^{-1}}{x_{g}^{-1}-x_{n}^{-1}}, & x_{n}^{-1}<x^{*}<x_{g}^{-1} \\
1, & x_{g}^{-1} \leq x^{*} \leq x_{g}^{+1} \\
\frac{x_{n}^{+1}-x^{*}}{x_{n}^{+1}-x_{g}^{+1}}, & x_{g}^{+1}<x^{*}<x_{n}^{+1} \\
0, & x^{*} \geq x_{n}^{+1}
\end{array}\right. \\
\mu_{z}\left(x^{*}\right)= \begin{cases}0, & x^{*} \leq x_{b}^{-1} \\
\frac{x^{*}-x_{b}^{-1}}{x_{n}^{-1}-x_{b}^{-1}}, & x_{b}^{-1}<x^{*} \leq x_{n}^{-1} \\
\frac{x_{g}^{-1}-x^{*}}{x_{g}^{-1}-x_{n}^{-1}}, & x_{n}^{-1} \leq x^{*}<x_{g}^{-1} \\
0, & x_{g}^{-1} \leq x^{*} \leq x_{g}^{+1} \\
\frac{x^{*}-x_{g}^{+1}}{x_{n}^{+1}-x_{g}^{+1}}, & x_{g}^{+1}<x^{*} \leq x_{n}^{+1} \\
\frac{x_{b}^{+1}-x^{*}}{x_{b}^{+1}-x_{n}^{+1}}, & x_{n}^{+1} \leq x^{*}<x_{b}^{+1} \\
0, & x^{*} \geq x_{b}^{+1}\end{cases}
\end{gathered}
$$




$$
\mu_{c}\left(x^{*}\right)=\left\{\begin{array}{lc}
1, & x^{*} \leq x_{b}^{-1} \\
\frac{x_{n}^{-1}-x^{*}}{x_{n}^{-1}-x_{b}^{-1}}, & x_{b}^{-1}<x^{*}<x_{n}^{-1} \\
0, & x_{n}^{-1} \leq x^{*} \leq x_{n}^{+1} \\
\frac{x^{*}-x_{n}^{+1}}{x_{b}^{+1}-x_{n}^{+1}}, & x_{n}^{+1}<x^{*}<x_{b}^{+1} \\
1, & x^{*} \geq x_{b}^{+1}
\end{array}\right.
$$

\section{Comprehensive Evaluation of Waterflooding Effect}

There are two index system for oil field waterflooding effect evaluation: Original reservoir state evaluation; controlled development level evaluation. Comprehensive evaluation result can be got be combining the two results. Here formula (5.7) is used.

\subsection{Fuzzy Evaluation of Vectors by Single Index}

For oil field waterflooding effect evaluation, the 16 indexes can be classified into three types of functions with fuzzy membership degree, such as (4.1)-(4.3). The standing points for good, normal, poor are needed when we calculatethe fuzzy evaluation vectors $V=\left\{\begin{array}{lll}h & Z & c\end{array}\right\}=\{$ good normal poor $\}$ of three blocks in $\mathrm{D}$ oil field, i.e. evaluation standards $x_{g}, x_{n}, x_{b}$ or $x_{g}^{+1}, x_{g}^{-1}, x_{n}^{+1}, x_{n}^{-1}, x_{b}^{+1}, x_{b}^{-1}$. This evaluation index can be got at least by three methods: Oil displacement experimental calculation, mechanism calculation of production fluid flow through porous media, statistical calculation. There are three blocks need to be evaluated, all have certain representativeness. For example, according to Table 4 and Table 5, the average value for 3 samples of each index is $x_{n}$, the average value for samples larger or smaller than $x_{n}$ is $x_{g}$ or $x_{b}$, etc. Others will not be listed here for sake of limited space.

Table 4. Data from original geological status.

\begin{tabular}{cccccccccc}
\hline blocks & Index1 & Ind2 & Ind3 & Ind4 & Ind5 & Ind6 & Ind7 & Ind8 & Ind9 \\
\hline block1 & 0.26 & 0.29 & 52.67 & 0.62 & 0.71 & 8.70 & 0.75 & 608.42 & 0.47 \\
block2 & 0.34 & 0.40 & 66.91 & 0.64 & 0.71 & 8.70 & 1.78 & 753.87 & 0.47 \\
block3 & 0.34 & 0.33 & 58.34 & 0.62 & 0.70 & 8.70 & 1.30 & 642.06 & 0.46 \\
\hline
\end{tabular}

Table 5. Control index data.

\begin{tabular}{cccccccc}
\hline blocks & Index1 & Index2 & Index3 & Index4 & Index5 & Index6 & Index7 \\
\hline block1 & -3.10 & 2.20 & 70.30 & 14.64 & 0.86 & 96.30 & 94.80 \\
block2 & -3.48 & 2.06 & 79.10 & 33.68 & 1.07 & 93.80 & 92.40 \\
block3 & -1.53 & 1.71 & 48.93 & 33.82 & 1.12 & 89.40 & 87.60 \\
\hline
\end{tabular}

For 16 indexes for comprehensive evaluation, there are 10 the larger the better 
indexes: channel sand proportion $R_{h d}$, net thickness $h_{e}$, initial oil saturation $S_{o}$, difference between reservoir pressure and saturation pressure $P_{b}$, reserves abundance $A_{q}$, displacement coefficient $\eta_{D}$, total pressure difference $\Delta P$, cumulative water storage rate $\gamma_{w}$, waterflooding controlling degree $C_{h}$, reserves recovery degree $D_{h}$. Single index fuzzy evaluation vector can be calculated by formula (4.1)-(4.3).

There are 4 better indexes: effective permeability $K_{e}$, coefficient of permeability variation $V_{K}$, oil viscosity $\mu_{o}$, cumulative injection production ratio $R_{r c}$. They can be obtained by formula (4.4)-(4.6).

There are two moderate indexes: oil water well ratio $R_{j}$, well spacing density $A_{j}$. For the two indexes, the rational oil and water well ratio and rational well spacing density of the three blocks should be calculated based on the technique, reservoir property, controlled development program, etc., of D oil field. Then use formula (4.7)-(4.9) to calculate.

The fuzzy evaluation vector of original reservoir geological property can be calculated by the above mentioned method, and it is signed as $r_{i j}^{k}(i=1,2, \cdots, 9 ; j=1,2,3 ; k=1,2,3)$, in it, subscript $i=1,2, \cdots, 9$ stands for 9 reservoir geological property indexes, subscript $j=1,2,3$ stands for good, normal, poor respectively, $k=1,2,3$ stands for number of 3 blocks.

Similarly, the fuzzy evaluation vector of the controlled development level can be calculated by the above function with fuzzy membership degree

$$
t_{i j}^{k}(i=1,2, \cdots, 7 ; j=1,2,3 ; k=1,2,3) .
$$

\subsection{Fuzzy Evaluation Vectors for Blocks}

The fuzzy evaluation result of each block is obtained by weighting the single index fuzzy vector. Synthetic operator $M(\bullet, \oplus)$ [17] is used; it is in the type of weighted average, putting emphasis both on weight and on the information of each evaluation index. Hence the fuzzy evaluation result is

$$
\tilde{D}^{k}=V \circ R^{k}=\left(v_{1}, v_{2}, \cdots, v_{9}\right) \circ\left(r_{i j}^{k}\right)_{9 \times 3}
$$

where $R^{k}=\left(r_{i j}^{k}\right)_{9 \times 3}$ is the evaluation result of $k$ block, synthetic fuzzy operator "。” is

$$
\left(v_{1}, v_{2}, \cdots, v_{9}\right) \circ\left(r_{11}^{k}, r_{21}^{k}, \cdots, r_{91}^{k}\right)^{\mathrm{T}}=\inf \left\{\sum_{i=1}^{9} v_{i} r_{i 1}^{k}, 1\right\}
$$

when calculating the fuzzy evaluation result of reservoir geological property of three blocks using formula (5.1), since the weight $V=\left(v_{1}, v_{2}, \cdots, v_{9}\right)^{\mathrm{T}}$ (formula (3.3)) and each factor in fuzzy matrix $R^{k}=\left(r_{i j}^{k}\right)_{9 \times 3}$ is smaller than 1 , and the sum of $R^{k}$ corresponding column factors is 1 , thus the use of synthetic fuzzy operator " $\circ$ " in formula (5.2) is the same with normal multiplication operator, then the fuzzy calculating formula (5.1) can be note as normal matrix multiplication, i.e.

$$
\tilde{D}^{k}=V^{\mathrm{T}} R^{k}=\left(v_{1}, v_{2}, \cdots, v_{9}\right)\left(r_{i j}^{k}\right)_{9 \times 3}
$$


Or to be signed $\tilde{D}=\left(d_{1}^{k}, d_{2}^{k}, d_{3}^{k}\right)$ (geological fuzzy evaluation vector), to $k=1,2,3$

$$
d_{i}^{k}=v_{1} r_{1 i}^{k}+v_{2} r_{2 i}^{k}+\cdots+v_{9} r_{9 i}^{k}, \quad i=1,2,3, k=1,2,3
$$

Similarly, the fuzzy evaluation vector for controlled development level can be got $\tilde{C}=\left(c_{1}^{k}, c_{2}^{k}, c_{3}^{k}\right)^{\mathrm{T}}$, among them

$$
c_{i}^{k}=w_{1} t_{1 i}^{k}+w_{2} t_{2 i}^{k}+\cdots+w_{7} t_{7 i}^{k}, \quad i=1,2,3, k=1,2,3
$$

The fuzzy evaluation result of three blocks calculated by using formula (5.3) and (5.4) is listed in Table 6.

\subsection{Convert Fuzzy Evaluation of Vector to a Number}

For the convenience of application and comparison, we need assign a real number for these fuzzy vectors. Fuzzy levels are assigned as "good, normal, poor", and the corresponding numerical values are " $100,50,0$ " respectively for better and direct understanding, so the numerical value for fuzzy comprehensive evaluation vector $\left(r_{1}, r_{2}, r_{3}\right)^{\mathrm{T}}=(\text { good, mediate, poor })^{\mathrm{T}}$ is

$$
Y=100 \times r_{1}+50 \times r_{2}
$$

Fuzzy evaluation of vectors of three blocks is determined by using formula (5.5) and listed in Table 6.

\subsection{Comprehensive Evaluation and Calculation}

The scores for original reservoir geological property and controlled development level of three blocks in D oil field are calculated by using formula (5.3)-(5.5), the result is listed in attached $\mathrm{C}$. The oil field waterflooding effect is mainly determined by controlled development level. Yet controlling development level depends greatly on original reservoir geological property. Therefore, the waterflooding effect can be defined as score for controlled development level divided by score for reservoir geological property, i.e.

$$
\begin{aligned}
& \text { score for comprehensive development effect } \\
& =\frac{\text { score for controlled development level }}{\text { score for reservoir geological property }}
\end{aligned}
$$

\begin{tabular}{|c|c|c|c|c|c|c|c|c|c|c|}
\hline \multirow{3}{*}{ block } & \multicolumn{4}{|c|}{ Reservoir geology evaluation } & \multicolumn{4}{|c|}{$\begin{array}{c}\text { Evaluation of controlled } \\
\text { development effect }\end{array}$} & \multicolumn{2}{|c|}{$\begin{array}{c}\text { Comprehensive } \\
\text { evaluation }\end{array}$} \\
\hline & \multicolumn{3}{|c|}{ fuzzy } & \multirow{2}{*}{ value } & \multicolumn{3}{|c|}{ fuzzy } & \multirow{2}{*}{ value } & \multirow{2}{*}{ value } & \multirow{2}{*}{ rank } \\
\hline & good & normal & poor & & good & normal & poor & & & \\
\hline 1 & 0.38 & 0.068 & 0.547 & 47.8 & 0.385 & 0.068 & 0.547 & 41.9 & 0.766 & 2 \\
\hline 2 & 0.17 & 0.218 & 0.609 & 75.1 & 0.174 & 0.218 & 0.609 & 28.3 & 0.626 & 3 \\
\hline 3 & 0.11 & 0.589 & 0.298 & 64.8 & 0.114 & 0.589 & 0.298 & 40.8 & 0.791 & 1 \\
\hline
\end{tabular}

Table 6. Comprehensive fuzzy evaluation of development effect of blocks.

In ideal condition, the formula bears "normal geological property (score 50) and normal control level (score 50)". Hence, in order to rationally evaluate the comprehensive effect, the above formula should be adapted to the following to cal- 
culate comprehensive score:

$$
Y_{x}=\frac{Y_{c}-\bar{Y}_{c}+50}{Y_{d}-\bar{Y}_{d}+50}
$$

Among them, $Y_{x}$, stands for score for comprehensive development effect;

$Y_{c}$, score for controlled development level;

$\bar{Y}_{c}$, score for controlled development level of three blocks in D oil field;

$Y_{d}$, score for original reservoir geological property;

$\bar{Y}_{d}$, score for original reservoir geological property of three blocks in $\mathrm{D}$ oil field.

Thus, the comprehensive development effect of each block can be fully demonstrated by the comprehensive score. The comprehensive score of three blocks in $\mathrm{D}$ oil field can be obtained by using formula (5.7), which can be sequenced and be listed in Table 6 .

\section{Conclusions and Discussion}

Based on score for original reservoir geological property obtained from formula (5.3) and (5.5), and score for controlled development level from formula (5.4) and (5.5), oil field waterflooding effect can be got after calculating the comprehensive effect score of each block area from formula (5.7). These scores can be comprehended as: development effect is mainly decided by two factors, one is original reservoir geological property, or the original oil production capacity of a block, this is the comprehensive score for reservoir property by fuzzy evaluation. The second one is if the controlled development is rational and scientific, this is the comprehensive score for controlled development level by fuzzy evaluation. Apparently, the better the original reservoir property, the more oil we can get, as long as the development is rationally and scientifically controlled. Even if the reservoir property is not very good, we can still obtain good results by rationally and scientifically making use of reservoir geology. Therefore, as defined in formula (5.7), it is reasonable to take the controlled development level score bears in reservoir property score as the "unified evaluation standard".

From the result (Table 6) of three blocks in D oil field by using fuzzy evaluation method, this comprehensive evaluation method is effective whose conclusion is relatively coincide with reality. Not only the method for evaluating oil field waterflooding effect is set up in this paper, but also the evaluation standards and methods for determining the standards are set up.

\section{Acknowledgements}

Research is partly supported by National Natural Science Foundation of China (Grant No. 12071218).

\section{Conflicts of Interest}

The authors declare no conflicts of interest regarding the publication of this paper. 


\section{References}

[1] Liu, C.L. and Xiao, W. (2010) Index System of the Water Flooding Development of Oil Fields and Its Structural Analysis. Petroleum Exploration and Development, 37, 209-213. https://doi.org/10.1016/S1876-3804(10)60037-7

[2] Shi, C.F., Wang, F.L., Xiao, W., et al. (2008) Analysis on Development System Structure of Water Flooding in Oil Field. Petroleum Geology \& Oilfield Development in Daqing, 27, 71-73. (In Chinese)

[3] Liao, H.W., Wang, F.Q., Xue, Z.T., et al. (2002) Reservoir Behavior Analysis Based on Large Scale System Method. Petroleum Journal, 23, 45-49. (In Chinese)

[4] Min, T.C. (1989) Waterflooding Effect Evaluation of L Reservoir in Laojunmiao Oil Field. Petroleum Journal, 10, 46-53. (In Chinese)

[5] Yang, Z.M., Zhang, Y.Z., Hao, M.Q., et al. (2006) Comprehensive Reservoir Evaluation Method in Low Permeability Oil Field. Petroleum Journal, 27, 64-67. (In Chinese)

[6] Wu, Z. and Peng, P.S. (1994) Recovery Degree Calculation and Treatment Effect Evaluation in Waterflooding Oil Field. Petroleum Journal, 15, 76-81. (In Chinese)

[7] Feng, Q.H., Lv, A.M., Yu, H.J., et al. (2004) A New Method for Evaluating Waterflooding Effect. Petroleum University Journal, 28, 58-60. (In Chinese)

[8] Wang, F.Q. and Xue, Z.T. (1998) Evaluating Waterflooding Effect by Using Systematic Analysis Method. Block Fault Oil and Gas Field, 5, 39-42. (In Chinese)

[9] Gao, X.J., Song, Z.Q., Cheng, Z.P., et al. (2003) Comprehensive Evaluation Method Affecting Waterflooing Effect of Sandstone Reservoir. Petroleum Exploration and Development, 30, 68-69. (In Chinese)

[10] Zhang, X.Z., Zhang, L.H., Xiong, Y., et al. (2005) Method for Evaluating High Water Cut Oil Field Development Effect and Its Application. Daqing Petroleum Geology and Development, 24, 48-50. (In Chinese)

[11] Song, Z.Q., Zhao, L., Wang, R.F., et al. (2004) Application of a Waterflooding Effect Evaluation Method in Liaohe Oil Field. Journal of Xi an Petroleum University (Natural Science Edition), 19, 17-22. (In Chinese)

[12] Wang, G.Y., Huang, X.Y. and Fu, Z.G. (2003) Fuzzy Comprehensive Judgment on Sa 0 Formation of Daqing Lasaxing Oil Field. Daqing Petroleum Geology and Development, 22, 22-24. (In Chinese)

[13] Wang, G.X., Xie, J.Y., Fan, J., et al. (2002) Evaluating Oil Field Waterflooding Effect Using Instantaneous Water Factor. Xinjiang Petroleum Geology, 23, 239-241. (In Chinese)

[14] Zhang, R. (1992) Evaluating Oil Field Waterflooding Effect Using Water Storage Rate Curve. Petroleum Exploration and Development, 19, 63-68. (In Chinese)

[15] Ji, B.Y. (2004) Collection of Study on Reservoir Engineering Theory and Method. Petroleum Industry Press, Beijing. (In Chinese)

[16] Yuan, Q.F., Chen, L.H., Ren, Y.L., et al. (2005) Oil Field Development Program Compiling Method. Petroleum Industry Press, Beijing. (In Chinese)

[17] Qiu, D. (1991) Systematic Analysis on Multi Index Comprehensive Evaluation. China Statistics Press, Beijing. (In Chinese)

[18] Hu, Y.H. and He, S.H. (2000) Comprehensive Evaluation Method. Science and Technology Press, Beijing. (In Chinese)

[19] Xie, J.J. and Liu, C.P. (2000) Application of Fuzzy Mathematical Method. Huazhong University of Science and Technology, Wuhan. (In Chinese) 